\title{
$\mathfrak{I} \mathfrak{n} \mathfrak{a} \mathfrak{l} \mathfrak{t}$
}

\section{Erites פeft.}

Tolitoi in Deutichland. (Dtto Sarnact.) ........... Seite 1

Die Berliner Freie $\mathfrak{B u ̈ h n e . ~ ( \Re o b e r t ~ S e i f e n t ) . ~ . ~ . ~ . ~}$

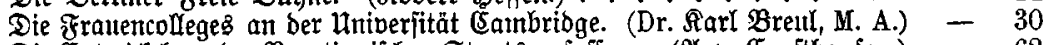

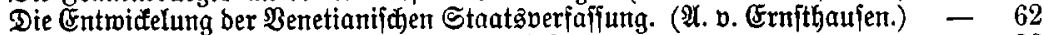

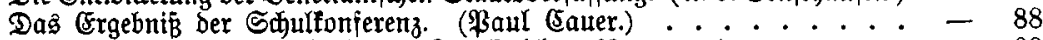

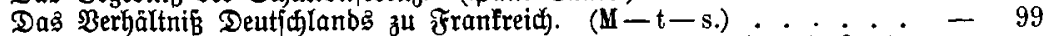

\$olitifue (Eorrefpondenz: Snntere \$olitif. Der Eonflict über bie Land: gemeinde=:Dromung. Die Sahulfonferenz. Sönig unb Miniter. (D.)

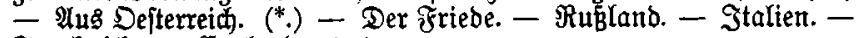
Franfreid. - England. (w.)

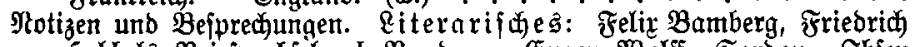
Sebbelă Briefmedjel. I. Band. - Eugen Molff, Sarbou, §bjen unb bie Bufunit bes beutfolt Drama's. - Dr. Sugo Raah, Die そrage Der Bolfäbülnen. - Rarl Retmbad, Zur Einfübrung in bas

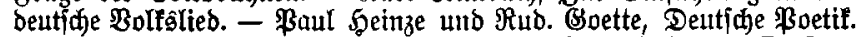

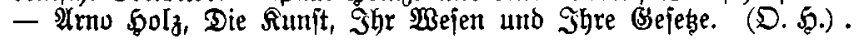

\section{3weites \$̧ejt.}

Plapoleon 1. und bie Suben. (Ernit Barre.) . . . . . . . . . . - 125

Goethes ELpenor. (Bujtav Settner.) . . . . . . . . . . . -149

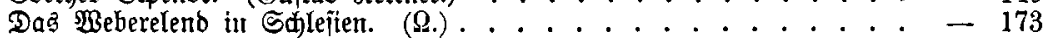

ฐ̧e

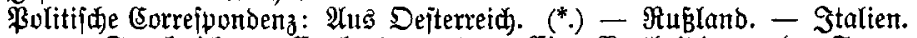
- Frantreid). - England. (w.) - Eine Bertheibigung ber Sperr:

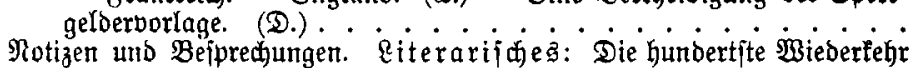

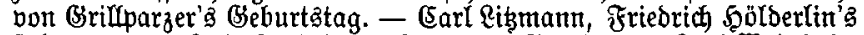
Reben. - Dr. Suh. Froibheim, Renz und Goethe. - Rarl Beinhold, Gebidte von $\mathfrak{S}$. M. R. Renz. - CE. Grifebad, Dả Goetheide Beitalter

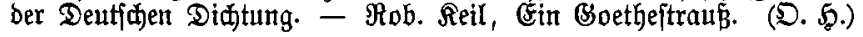

\section{Drittes Syeft.}

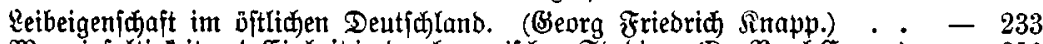

Pannigfaltigteit unb Einkeit in Den homerifden Stubien. (Dr. ßaul (Eauer.) - 250

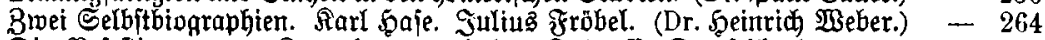

Die Befeftigung von Sopenthagen und bas Sntereffe Deutidhlands... - 279

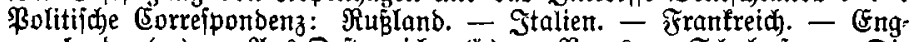

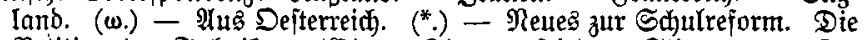
Petition ber Tedinifer. (E.) - Die unzufrtebene Stimmung. (D.)

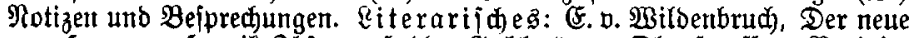

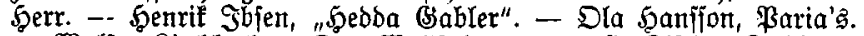

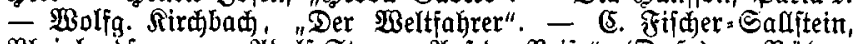

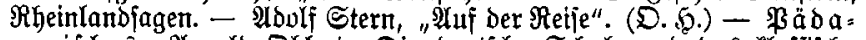

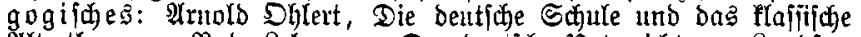

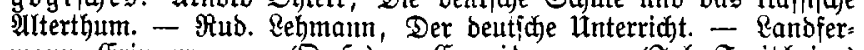
mann, (Erinnerungen. (‥5.) - Eriviberung. (Joh. Froibheim.)

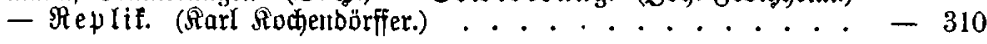

\section{Biertes \$̧eft.}

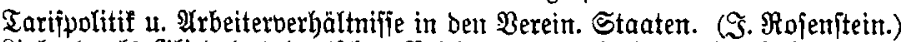

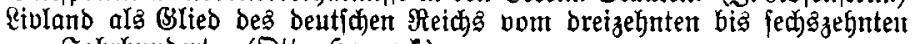
Jahrhumbert. (Dtto Şarnaf.) ............ - 364

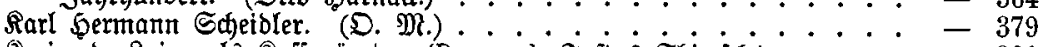

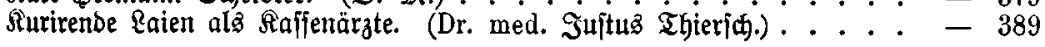




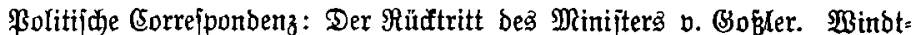

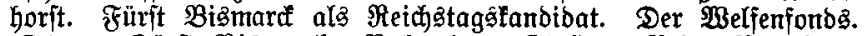
(D.) - Fürit Bismarff. Bulgarien. Itatien. Brinz Rapoleon.

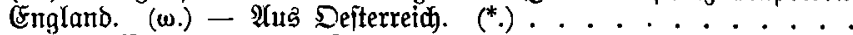

Notizen und Befpredungen. Riterarijols: B. Şauptmann, Einiame Deniden. - $\mathfrak{B}$. Sarbou, Thermibor. - Freberi Miftral, Nerto, Brobençalijche Erzählung, überi. v. $\mathfrak{A}$. Bertuch. - Johant Rudwig Runeberg's (Epifde Didutungen. Ueberf. b. $\mathfrak{B}$. Eigentrobt. 2 Th.

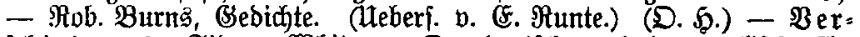
jhiebenes: Sibnen $\mathfrak{B h i t m a n}$, Der beutjhe und ber englijwe $\mathfrak{A r}=$

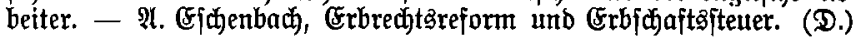

\section{Fünftes פeft.}

Das Ende des Traums. I. (George Duruy.). . . . . . . . - 421

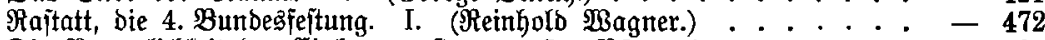

Die Berveglidffeit ber (Einfonmenftener. (Dr. Bünger.) . . . . . . - 499

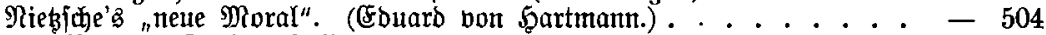

Die Berliner Runftausftellung. . . . . . . . . . . . . . . . -522

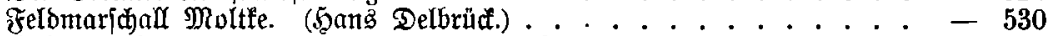

Bolitifhe (Eorrejpondenz: Der Mord in Sofia. Bur rujitichen \$olitif unb Finanzgeidichte. Die $\mathfrak{D}$ lorbe in $\Re$ lew $=$ Drleana. Die Explofiton it $\Re$ om. Der internationale $\mathfrak{A}$ rbeitercongres in Paris und bie bel=

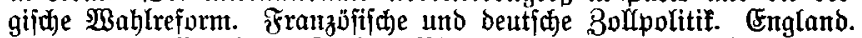
(w.) - Die $\mathfrak{B a g h l}$ deș Füriten Bis̄mardf. Die Stenergejebe. (D.)

Rotizen und Befpreduthen. Riterarifides: Die Rebaftion. - Arbert

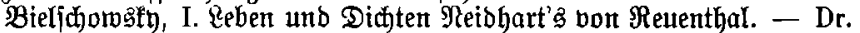

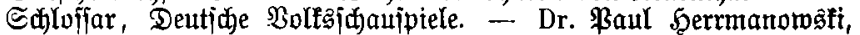
Die beutide (sijtterlehre. - Dtto Sdfroeder, Bom papiernen Stif.

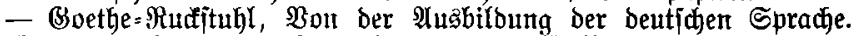

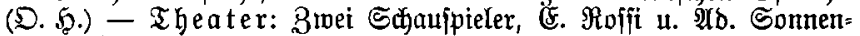

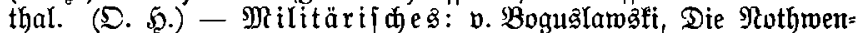
bigfeit der zmeijährigen Dientzeit. - Bronjart $\mathfrak{v}$. Safjellendorff I., Betrachtungen î̉ber eine zettgemäß̈e Fechtweife ber Snfanterie. Batid), Momiral \$rinz 2 doalbert von \$reufen. - \$rof. D. Frife, A

(D.)

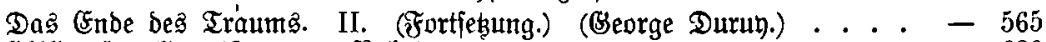

Ehilbe Sarold. (5ermann Balz.) . . . . . . . . . . . . -620

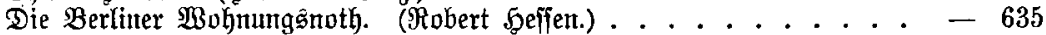

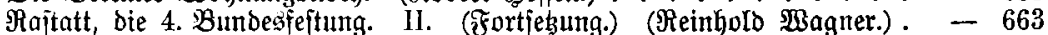

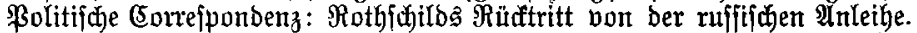

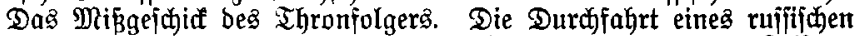

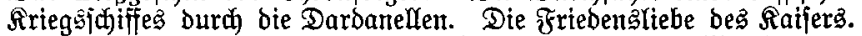

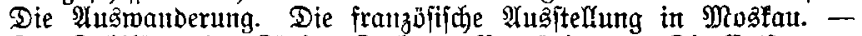
Das Subiläum bes Rünigs Sarl don Rumänien. - Die Entfermung ber Rünigin Ratalie aus Serbien. - Die Maifeter ber Sozialdemo=

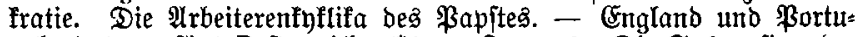

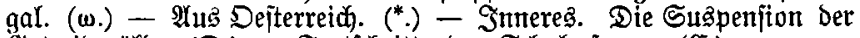

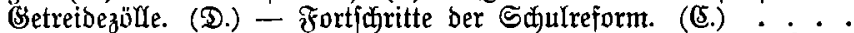

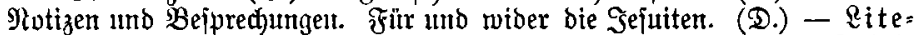
rarifa)

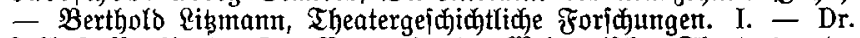

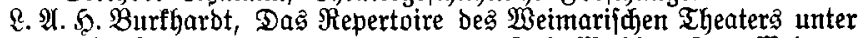
(3oethe's Reitunt 1791-1817. - Dr. Jul. $\mathfrak{B a h l e , ~ D a \Xi ~ M e t m a r . ~}$

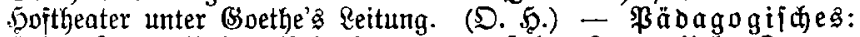
5̧ełene Eange, Utrjere Bejtrebungent. - Selene Range, Heber Frauen:

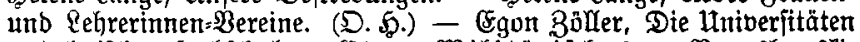

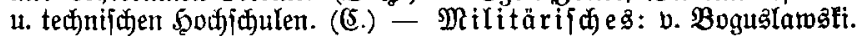

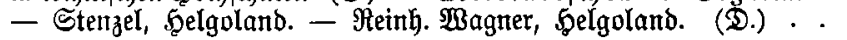

